\title{
EVALUACIÓN DE LA PERCEPCIÓN DEL ATRACTIVO DE LA SONRISA Y SU INFLUENCIA EN LA VALORACIÓN DE LAS CARACTERÍSTICAS PERSONALES:PRUEBA PILOTO
}

\section{EVALUATION OF THE PERCEPTION OF THE ATTRACTIVENESS OF THE SMILE AND ITS INFLUENCE IN THE EVALUATION OF THE PERSONAL CHARACTERISTICS: A PILOT TEST}

\author{
Ríos Villasis Katty ${ }^{1 *}$, Hidalgo Lostaunaun Rony ${ }^{2}$, Villavicencio Caparó Ebingen ${ }^{3}$ \\ ${ }^{1}$ Docente de la Maestría en Estomatología y con Mención, UPCH. Egresada del doctorado en Estomatología, UPCH. \\ Magíster en Estomatología, UPCH. Perú \\ ${ }^{2}$ Especialista en Odontología Restauradora y Estética, UCS. Profesor del postgrado en Rehabilitación Oral, UPSJB. \\ Coordinador del diplomado en Odontología Restauradora y Estética, UNMSM. Perú \\ ${ }^{3}$ Docente del departamento académico de Odontología Social, UPCH.Perú \\ *liz.rios.v@upch.pe
}

\section{Resumen}

OBJETIVO: Evaluar la percepción del atractivo de la sonrisa y su influencia en la valoración subjetiva de la competencia social, capacidad intelectual y equilibrio psicológico. Materiales y Métodos: Se realizó un estudio exploratorio, descriptivo, transversal; en dos etapas: La primera, consistió en una encuesta online a 25 odontólogos acerca de las características estéticas de la sonrisa; y en la segunda, se hicieron encuestas a 26 personas, donde se pidió a cada participante que juzgara sobre las características personales de una sola fotografía a color. Se empleó una fotografía de una mujer con alteraciones en la exposición gingival ( $\mathrm{EG}+4 \mathrm{~mm}$ ) y corredor bucal (CB amplio). Se usó la prueba de Anova para muestras independientes para comparar la media de los distintos grupos. Resultados: Los participantes valoraron a la persona fotografiada con un valor promedio de agradabilidad de la sonrisa de $8.01 \pm 1.08 \mathrm{~cm}$; así como, más competente desde un punto de vista social, con buen rendimiento intelectual y equilibrio psicológico. Conclusión: El atractivo de la sonrisa influye en los juicios que realiza una persona sobre las características personales de otro.

Palabras clave: Estética, sonrisa, personalidad, percepción.

Abstract

OBJETIVO: To evaluate the perception of the attractiveness of the smile and its influence in the subjective evaluation of the social competence, intellectual capacity and psychological balance. Materials and methods: An exploratory, descriptive, transverse study was carried out; In two stages: The first consisted of an online survey of 25 dentists about the aesthetic characteristics of the smile; And in the second, 26 people were surveyed, where each participant was asked to judge on the personal characteristics of a single color photograph. A photograph of a woman with alterations in the gingival exposure ( EG+ 4mm) and buccal corridor(CB amplio). was used. The Anova test for independent samples was used to compare the mean of the different groups. Results: The participants assessed the person photographed with the alteration of the two aesthetic parameters studied, with an average value of pleasantness of the smile of 8.01 \pm 1.08 ; As well as, more competent from a social point of view, with good intellectual performance and psychological balance. Conclusion: The judgments that one person makes on the personal characteristics of another, are influenced by the attractiveness of the smile; However, does not coincide with the appreciation of the dentist.

Key words: Aesthetics, smile, personality, perception. 


\section{INTRODUCCIÓN}

La preocupación de la gente por la belleza y el atractivo de sus dientes comenzó hace más de 2.000 años. ${ }^{1}$ La definición cultural de la belleza relacionada a la sonrisa difiere en las poblaciones, regiones, países e incluso continentes; además, de ser un concepto dinámico con parámetros que van cambiando por diversas razones a través del tiempo. ${ }^{2}$ En los últimos años, la preocupación por la odontología estética ha aumentado; esto se debe a la exposición de los medios de comunicación, y al paralelismo de la información; a través, de los cuales las personas pueden ver modelos de sonrisa como referentes y contar con información de los tratamientos de moda; ${ }^{2}$ Además, el atractivo de la sonrisa juega un papel clave en las interacciones sociales; ya que, ésta influye en el éxito de las relaciones, en la imagen profesional, en las oportunidades de empleo; y en el desarrollo de la personalidad. ${ }^{3}$ Pithon et al. ${ }^{4}$ evaluaron si la estética dental influye en la búsqueda de un nuevo empleo, encontrando que las personas con sonrisas ideales son consideradas más inteligentes y tienen una mayor probabilidad de encontrar un puesto de trabajo en comparación con las personas con sonrisas no ideales. En nuestra sociedad consciente de la belleza, las personas a menudo son juzgadas; y, por lo tanto, se juzgan así mismos por su sonrisa; la cual es alterada por las enfermedades de la boca que conlleva a una pérdida de la salud, la función y la estética ${ }^{5}$ impactando en la autoestima y la calidad de vida. ${ }^{6}$ Diversos estudios se han conducido para evaluar la percepción de la sonrisa alterando los parámetros estéticos que la componen, tales como, forma y color de los dientes anteriores, ${ }^{7,8}$ exposición gingival, ${ }^{9}$ arco de la sonrisa, corredores bucales, ${ }^{10}$ línea de la sonrisa, ${ }^{11}$ entre otros; que fueron valorados por odontólogos generales, especialistas y personas comunes para conocer la coherencia de las expectativas de estos grupos; sin embargo, quedó demostrado que tenían diferentes percepciones de la estética de la sonrisa. ${ }^{2}$ Una sonrisa ideal puede no existir, pero el objetivo estético más importante es lograr una sonrisa que armonice con la apariencia física y personalidad del paciente. Las expectativas estéticas de la mayoría, no pueden ser satisfechas solo aplicando las reglas de la estética natural de la sonrisa porque tales deseos en su mayoría están influenciados por el sexo, raza y/o factores de edad, pero generalmente se orientan por tendencias, cultura e intereses personales. Por lo tanto, una comprensión de la percepción estética de los parámetros que ayudan o perjudican una sonrisa son importantes para crear sonrisas atractivas; así como, conocer la influencia de ésta en la valoración subjetiva de la competencia social, habilidad intelectual y equilibrio psicológico.

\section{MATERIALES Y MÉTODOS}

Se realizó un estudio exploratorio, descriptivo y transversal; el cual se llevó a cabo en dos etapas: En la primera etapa, se realizó una búsqueda electrónica en Medline a través de Pubmed con la finalidad de determinar los parámetros más referidos en el atractivo de la sonrisa. Las palabras claves utilizadas para la búsqueda fueron: «Smile attractiveness», «Smile perceptions», «Personality», «Expectations», «Esthetics». Se identificaron catorce parámetros: Color dental, presencia de diastemas, exposición gingival, corredor bucal, línea media, tamaño dental, alineamiento dental, superficie dental, arco de la sonrisa, estética gingival, halo de los dientes, plano oclusal, triángulos negros y troneras inciales. Posteriormente, en la red social Facebook, se publicó la siguiente pregunta: ¿Qué características de la estética de la sonrisa consideras más relevantes?; dirigido a odontólogos generales y especialistas. Se obtuvieron un total de 25 respuestas entre comentarios y mensajes internos; de los cuales se obtuvo lo siguiente (Figura $\mathrm{N}^{o} 1$ ).

En la segunda etapa, se eligieron dos de los parámetros referidos dentro de los más relevantes; que fueron la exposición gingival y el corredor bucal. Se utilizó la fotografía de una mujer, la cual previamente fue notificada con el propósito del estudio. Una vez que aceptó participar en el estudio voluntariamente, firmó un consentimiento informado. Se le tomaron varias fotografías utilizando una cámara Nikon D3100 apoyada sobre un trípode, contra una pared de fondo blanco a una distancia de $30 \mathrm{~cm}$ y a $1 \mathrm{~m}$ de la cámara con el rostro mirando al frente durante la sonrisa y el plano de Frankfort paralelo al piso. La instrucción fue tener el cabello amarrado, no usar aretes, no tener maquillaje, no cerrar los ojos, humedecerse los labios y sonreír. Luego de que un juicio de expertos seleccionó la fotografía más adecuada; esta fue modificada por un experto en computación mediante el programa Adobe Photoshop CC2014, obteniendo una fotografía con una exposición gingival (EG) de $+4 \mathrm{~mm}$ conjugada con un corredor bucal (CB) amplio (Figura $\mathrm{N}^{o} 2$ ).

La fotografía modificada fue impresa para su posterior análisis. Se encuestaron a 26 personas; a quienes se les explicó el propósito del estudio y luego que aceptaron participar voluntariamente en la investigación se les entregó el cuestionario indicándole que podían evaluarlo por un periodo máximo de 1 min y valorarlo mediante la Escala Visual Análoga (EVA). ${ }^{12-15}$ La encuesta incluyó información como: sexo y edad. La escala visual análoga estuvo compuesta por una línea recta de $10 \mathrm{~cm}$ de longitud que iba de 0 a 10 donde cero es "no me agrada" y 10 "me agrada mucho". Para las apreciaciones de los participantes acerca de la persona fotografiada se utilizó un cuestionario ${ }^{16,17}$ que constaba de tres categorías: la competencia social (CS), la capacidad intelectual (CI) y el equilibrio psicológico (EP); dividido en nueve apartados, tres para cada característica:

CS1: Se les pidió que puntuaran la popularidad que creían que tenía dicha persona entre sus compañeros. Las puntuaciones se llevaron a cabo sobre una escala de Likert de cinco puntos, cuyos extremos fueron «muy popular) y «muy impopular». CS2: Se pidió a los participantes que determinaran lo amigable que parecía la persona. Las respuestas se 


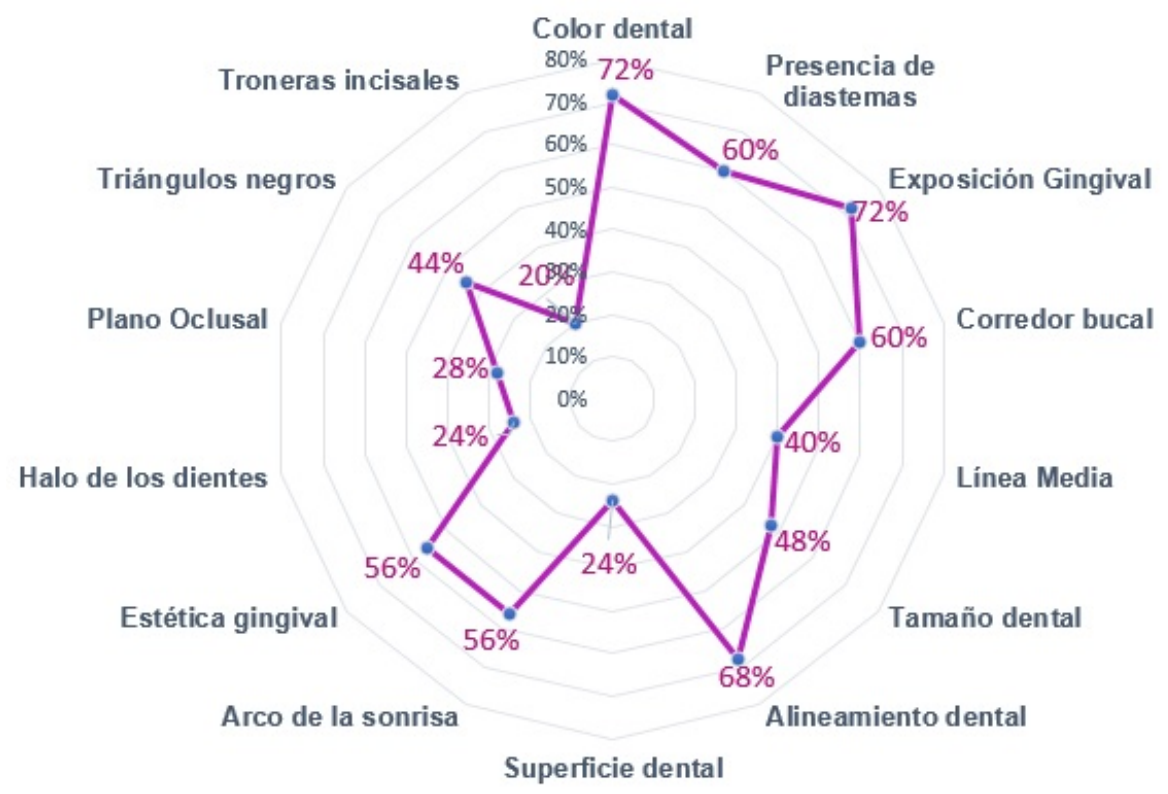

Fig. 1. Características de la estética de la sonrisa consideradas más relevantes.
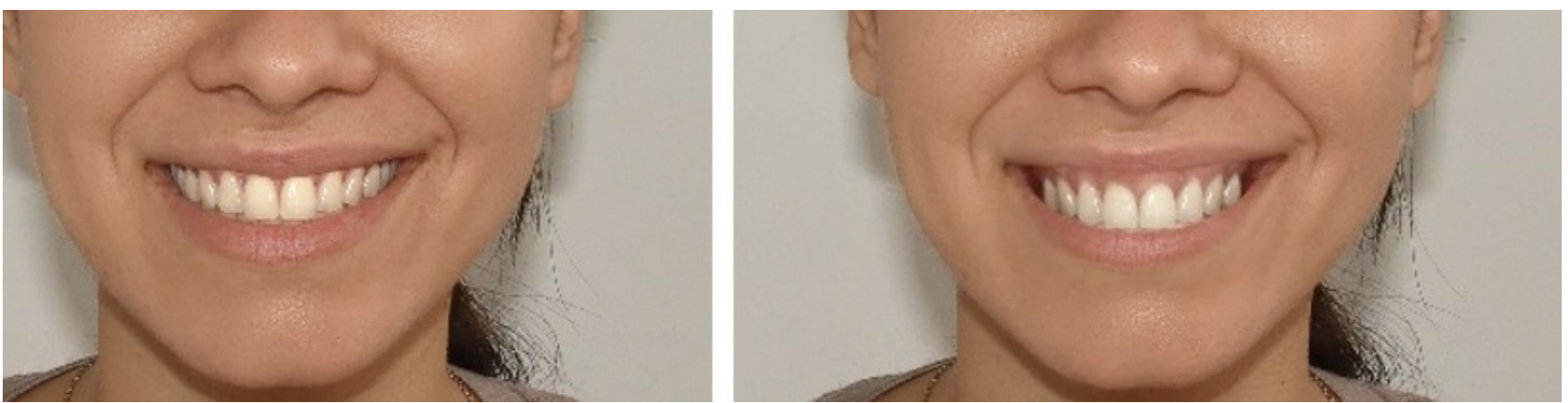

Fig. 2. Modificación de la fotografía

realizaron sobre una escala de Likert que iba desde «muy amigable» a «muy poco amigable». CS3: Se pidió a los participantes que indicaran si creían que la persona poseía una buena vida social. Las opciones de respuesta fueron «sí», «no», y «no sé». . Cl1: Los participantes debían puntuar el grado de éxito conseguido en la escuela por la persona de la fotografía. Las respuestas se daban sobre una escala de Likert situada entre «mucho éxito» y «muy poco éxito». . Cl2: Se pidió a los participantes que puntuaran la inteligencia que aparentaba la persona sobre una escala de Likert de cinco puntos, que abarcaba de «muy inteligente» a «muy poco inteligente». . C13: Se preguntó a los participantes si pensaban que la persona había ido a la universidad o no. Las opciones de respuesta fueron «sí», «no» y «no sé». EP1: Se les pidió que puntuaran si creían que la persona era extrovertida o introvertida sobre una escala de Likert de cinco puntos, que iba desde «muy introvertida» a «muy extrovertida». . EP2: El grado de felicidad que transmitía la persona fue puntuado sobre una escala de Likert de cinco puntos, que abarcaba desde «muy feliz» a «muy infeliz». .. EP3: Se pidió a los pacientes que puntuaran el grado de autoestima de la persona sobre una escala de Likert de cinco puntos, que fuera de «mucha autoestima» a «ninguna autoestima». Las puntuaciones se obtuvieron de las tres escalas sumando las valoraciones de cada apartado. Una vez que se recolectaron todas las encuestas, los resultados fueron digitados y codificados en una hoja de cálculo de Excell y procesados en el paquete estadísticos SPSS vs. 21; para lo cual se fijó un nivel de significancia del $5 \%$. En la estadística descriptiva se obtuvieron medias, desviación estándar, mínimo y máximo; y para la estadística 
analítica utilizó las pruebas de U- de Mann Whitney y Anova, Tukey.

\section{RESULTADOS}

En el estudio participaron un total de 26 personas, el $42,3(\mathrm{n}=11)$ pertenecientes al sexo masculino y el $57,7 \%$ $(\mathrm{n}=15)$ al sexo femenino; con edad promedio de 37,69+ 13,81 años. Al evaluar la percepción estética de la fotografía modificada mediante la escala visual análoga (EVA), se obtuvo un valor promedio de 7,9+1,29 cm. Mientras que, al evaluar la percepción de la sonrisa según sexo del evaluador se obtuvo un valor promedio de $8,09 \pm 1,59 \mathrm{~cm}$ en el sexo masculino y $7,79 \pm 1,13 \mathrm{~cm}$ en el sexo femenino. No se encontraron diferencias significativas entre el valor promedio de la percepción estética de la sonrisa y el sexo (Prueba de U de Mann-Whitney; $p>0,05)\left(\right.$ Tabla $\left.\mathrm{N}^{o} 1\right)$

Tabla 1. Percepción estética de la sonrisa según la escala visual análoga, de acuerdo al sexo.

\begin{tabular}{llllll}
\hline \multicolumn{5}{c}{ Percepción estética $(\mathrm{cm})$} \\
Sexo & $\mathrm{x}$ & D.S. & Mín. & Máx. & Valor $\mathrm{p}$ \\
\hline Masculino & 8,09 & 1,53 & 4,50 & 10,00 & $\mathrm{p}>0,05^{\star}$ \\
Femenino & 7,79 & 1,13 & 5,00 & 9,00 & \\
\hline
\end{tabular}

En cuanto a la valoración de la característica personales, se obtuvo que los evaluadores consideraron a la persona de la fotografía evaluada un $53,8 \%$ popular; un $69,2 \%$ amigable; un $80,8 \%$ que posee una buena vida social; un $53,8 \%$ que tiene un grado de éxito moderado; un $88,5 \%$ lo considera inteligente; un $80,8 \%$ cree que ha estudiado una carrera universitaria; un $76,9 \%$ la considera como una persona extrovertida; un $80,8 \%$ cree que trasmite felicidad y le dan un $76,9 \%$ de mucha autoestima (Tabla $\mathrm{N}^{\circ} 2$ ).

$\mathrm{Al}$ evaluar las dimensiones del cuestionario, que constaba de tres categorías: la competencia social (CS), la capacidad intelectual (CI) y el equilibrio psicológico (EP); se obtuvo que la dimensión con mayor puntuación fue la de equilibrio psicológico con 11,85; mientras que las dimensiones de competencia social y capacidad intelectual obtuvieron una puntuación de 10,54 y 10,31; respectivamente. Se encontró diferencia estadísticamente significativa entre las dimensiones de las características personales valoradas (Prueba Anova de Tukey; $\mathrm{p}<0,05)\left(\right.$ Tabla N$\left.^{o} 2\right)$.

\section{DISCUSIÓN}

Las anomalías dentales visibles influyen en la apreciación que los individuos hacen en relación con las características de una persona. ${ }^{12}$ Se ha considerado que la edad y el sexo, entre otros factores, influyen en las percepciones estéticas de las personas sobre el atractivo de la sonrisa.
Tabla 2. Percepción de las características personales de la fotografía evaluada.

\begin{tabular}{ll}
\hline $\begin{array}{l}\text { Características } \\
\text { valoradas }\end{array}$ & personales \% \\
\hline Popular & 63,8 \\
Amigable & 69,2 \\
Buena vida social & 80,8 \\
Moderado éxito & 53,8 \\
Inteligente & 88,5 \\
Fue a la universidad & 80,8 \\
Extrovertido & 76,9 \\
Feliz & 80,8 \\
Mucha autoestima & 76,9 \\
\hline
\end{tabular}

Tabla 3. Percepción de la competencia social, capacidad intelectual y equilibrio psicológico de la fotografía evaluada.

\begin{tabular}{llllll}
\hline & $\mathrm{x}$ & D.S. & Mín. & Máx. & \\
& & & & & Valor $p$ \\
\hline Competencia Social & 10,54 & 1,39 & 7,00 & 13,00 & \\
Habilidad Intelectual & 10,31 & 0,88 & 9,00 & 12,00 & $p<0,05^{*}$ \\
Equilibrio Psicológico & 11,85 & 1,01 & 10,00 & 14,00 & \\
*Anova, Tukey & & & & &
\end{tabular}

Algunos estudios han sugerido que el color de los dientes es un factor significativo en el atractivo de la sonrisa. ${ }^{13-15}$ En nuestro estudio, se realizó primeramente una recopilación de la literatura científica en cuánto a los parámetros que influyen en el atractivo de la sonrisa; y posteriormente, se hizo una evaluación exploratoria con la finalidad de conocer la jerarquía de estas características (Figura $\mathrm{N}^{o} 1$ ). Todos los participantes coinciden en muchos de los parámetros estéticos reportados por la literatura; $;{ }^{13-15}$ aunque, según la especialidad hay una jerarquía de los parámetros que podrían definir para cada uno una sonrisa ideal; en base a esto, y en la mayoría de los casos no se deberá entender como fracaso no llegar al concepto ideal si se ha logrado una sonrisa bella, armónica y sobretodo saludable, aunque ésta no reúna todas las características. En este estudio, se evaluó la combinación de dos de las características consideradas dentro de las más relevantes; que fueron la EG y el CB para obtener información sobre la influencia que ejercen estos parámetros sobre la percepción estética de la sonrisa en nuestra población; así como, en la valoración de las características personales. Se sabe que las anomalías dentales visibles influyen en la apreciación que los individuos hacen en relación con las ca- 
racterísticas de una persona; la presencia de dientes careados o con alteraciones del color son asociados con niveles más bajos de competencia social e intelectual y con un menor equilibrio psicológico; sin embargo, no existen reportes de otras alteraciones dentales consideradas como relevantes para el facultativo y no tan visible para el paciente; por ello, se decidió alterar la fotografía de la modelo con variaciones de una EG+4mm, elegida como la sonrisa menos agradable cuando fue evaluada por ortodoncistas, odontólogos y personas comunes; según lo reportado por Brum et al. ${ }^{16}$; junto con un $\mathrm{CB}$ amplio; también considerado como el menos agradable en el estudio de Ritter et al. ${ }^{17}$ En la segunda etapa de este estudio; cuando las personas valoraron la percepción de la sonrisa de las variaciones anteriormente mencionadas con la escala visual análoga (EVA); le atribuyeron un valor promedio alto de $8,09 \pm 1,59 \mathrm{~cm}$; siendo está puntuación dada, mayor en el sexo masculino en comparación al sexo femenino; sin diferencias significativas entre ambos grupos. $\mathrm{Al}$ evaluar las características personales de la persona evaluada; se le atribuyeron juicios tales como: un $88,5 \%$ la considera inteligente, un $80,8 \%$ creen que posee una buena vida social, que ha estudiado una carrera universitaria y que transmite felicidad; seguido de un $76,9 \%$ que la considera como una persona extrovertida y con mucha autoestima; y finalmente, un $53,8 \%$ considera que tienen popularidad y un grado de éxito moderado. Cuando se analizaron estas valoraciones según sus dimensiones, se encontró una mayor puntuación en la dimensión de equilibrio psicológico; seguido de la competencia social y capacidad intelectual; con diferencias estadísticamente significativas entre las puntuaciones de las dimensiones. Esto coincide con lo evaluado en el cuestionario; ya que las respuestas dadas según la escala de Likert; dieron mayor puntuación a que la persona tiene mucha autoestima, es una persona extrovertida y transmite felicidad; ítems que componen la dimensión de equilibrio psicológico. Las conclusiones extraídas de estos resultados deben considerar las limitaciones del estudio; ya que, la situación llevó a los participantes a hacer juicios arbitrarios; sin embargo, las respuestas en base a la valoración de la fotografía mostraron un patrón consistente. Los participantes no dieron respuestas aleatorias, sino que las mismas fueron influidas por las alteraciones realizadas en la sonrisa. Es posible que los individuos para los que la salud oral tiene alguna importancia sean más proclives a evaluar el atractivo de la sonrisa como elemento para emitir juicios acerca del equilibrio psicológico y la competencia intelectual; así mismo, esta información es valiosa ya que poner en evidencia que lo que el facultativo percibe difiere de lo que el paciente percibe; por ello, debemos ser asertivos buscando un punto de equilibrio justo entre la necesidad sentida y expresada de nuestros pacientes y la necesidad normativa propia de nuestra formación como profesionales. Las nuevas definiciones de la salud oral no sólo se refieren a la ausencia de enfermedad, sino al rol que juega la salud, función y estética de la sonrisa en la determinación del bienestar social y psicológico del individuo. Es menester, considerar el uso de un protocolo básico multidisciplinario que sirva dentro de un plan de tratamiento en el que además de la función se tenga en cuenta la obtención de resultados que cumplan con las demandas estéticas y expectativas de nuestros pacientes.

\section{CONCLUSIONES}

El atractivo de la sonrisa influye en los juicios que realiza una persona sobre las características personales de otro; sin embargo, la percepción de las características consideradas relevantes en la estética de la sonrisa por los facultativos difiere de lo que perciben las personas comunes.

Conflicto de intereses y financiamiento Los autores declaran no tener conflicto de intereses, haber cumplido con los requisitos de autoría y haber autofinanciado este artículo.

\section{Referencias}

1 Rodrigues C, Magnani R, Machado MS, Oliveira OB. The perception of smile attractiveness. Angle Orthod. 2009;79(4):634-639.

2 Mokhtar H, Abuljadayel L, Al-Ali R, Yousef M. The perception of smile attractiveness among Saudi population. Clinical, Cosmetic and Investigational Dentistry 2015; 7: 17-23.

3 Van der P, osterveld P, Van G, Kuijpers AM. Smile Attractiveness: Self-perception and Influence on Personality. Angle Orthodontist 2007; 77 (5):759-65.

4 Pithon M, Nascimento C, Gouveia G, Coqueiro R. Do dental esthetics have any influence on finding a job?. Am J Orthod Dentofacial Orthop 2014;146(4):423-9.

5 Rehman K, RazaKhan F, Rahman M. Assessing the perception of smile attractiveness in young adults: A pilot study.

6 De Paula J, Santos N, Da Silva E, Nunes M, Leles C. Psychosocial Impact of Dental Esthetics on Quality of Life in Adolescents. Angle Orthodontist. 2009; 79(6): 1188-93.

7 Murthy S, Ramani N. Evaluation of natural smile: Golden proportion, RED or Golden percentage. J Conserv Dent. 2008; 11(1): 16-21.

8 Heravi F, Rashed R, Abachizadeh H.Esthetic preferences for the shape of anterior teeth in a posed smile. AJODO. 2011; 139(6): 806-14.

9 Gracel F, Pinho T. Assessment of the perception of smile esthetics by laypersons, dental students and dental practitioners. International Orthodontics. 2013; 11(1): 432-44.

10 Springer N, Chang C, Fields H, Beck M, Firestone A, Rosenstiel S. Smile esthetics from the layperson's perspective. AJO-DO. 2011; 139(1): e91-101.

11 Van der Geld P, Oosterveld P, Schols J, Kuijpers-Jagtmand A. Smile line assessment comparing quantitativemeasurement and visual estimation. Am J Orthod Dentofacial Orthop. 2011; 139(2):174-80. 
12 Thimothy J, Prabhu N, Robinson P. The Impact of Dental Appearanceon the Appraisal of Personal Characteristics. Int J Prosthodont. 2003;16(4):429-34.

13 Kihn PW. Vital tooth whitening. Dent Clin North Am. 2007;51(2):319-331, viii.

14 Vivek S, Vikas P, Meenakshi K, Sandhya P, Rao Lakshmana B. A study of relationship between skin color and tooth shade value in Population of Udaipur, Rajasthan. International Journal of Dental Clinics. 2010;2(4):26-29.

15 Rehman K, Khan FR, Rahman M. Assessing the perception of smile attractiveness in young adults: A pilot study. J Pak Dent Assoc. 2011;20(4):206-210.

16 Brum M, Enéas D, Borgatto A, D’Agostine C, Rocha R. Influência da exposição gengival na estética do sorriso. Dental Press J Orthod. 2011; 16(5):111-8.

17 Ritter DE, Gandini LG, Pinto Ados S, Locks A. Esthetic influence of negative space in the buccal corridor during smiling. Angle Orthod. 2006; 76(2):198-203.

Recibido: 10 de Febrero de 2017

Aceptado: 15 de Marzo de 2017 